Jacques Dubois et Pascal Durand, Université de Liège.

\title{
CHAMP LITTÉRAIRE ET CLASSES DE TEXTES
}

"L'objet d'art - comme tout autre produit - crée un public sensible à l'art, un public qui sait jouir de la beauté. La production ne crée donc pas seulement un objet pour le sujet, mais aussi un sujet pour l'objet. Elle produit donc la consommation a) en lui fournissant la matière; b) en déterminant le mode de la consommation; c) en faisant naître chez le consommateur le besoin de produits qu'elle a d'abord posés sous forme d'objets. Elle crée par conséquent l'objet, le mode et l'instinct de la consommation. De même la consommation produit le talent du producteur en le sollicitant en tant que besoin mû par une finalité."

Karl MARX'.

\section{Un concept déclassé}

Comme les hommes et comme les théories qui les produisent, les concepts sont obsolètes et mortels. Certains, naguère en faveur, sont évincés par de nouveaux que la mode ou la commodité imposent; d'autres sont frappés peu à peu de discrédit, quand ils ne tombent pas sous le coup d'un interdit. Ainsi en va-t-il aujourd'hui du concept de "classe sociale" qui semble exclu du discours général et ne fait plus guère, dans le discours même qui le forgea la sociologie -, que des apparitions discrètes, aussitôt nimbées de multiples précautions. Sans doute voit-on bien l'enjeu idéologique qui préside à pareil

1. Introduction générale à la critique de l'économie politique (1857), dans Guvres, Économie I, Paris, Gallimard, Bibliothèque de la Pléiade, 1965, p. 245 (Traduction par M. Rubel et L. Evrard). 
refoulement (supprimer le mot pour dénier ce qu'il représente) et de quel contexte sociopolitique celui-ci relève (une société où le mirage libéral de la "mobilité » estompe les clivages et les antagonismes de classes); il n'en reste pas moins que le déclin du concept participe aussi de notre moderne défiance envers les divisions schématiques et les visions prophétiques héritées du siècle dernier. La grande et ultime confrontation des classes n'aura pas lieu, du moins pas dans les cadres de la prédiction marxienne. Et si la lutte continue, elle ne se manifeste généralement plus que de manière sournoise ou détournée. Les rapports de production et de domination se font de plus en plus abstraits et obliques cependant que la simple appartenance à telle fraction sociale, dans une société mouvante - la nôtre -, est devenue plus que jamais un fait complexe et diffus. En sorte qu'on en vient (trop) aisément à penser que les classes n'existeraient qu'à titre d'effets glissants à la surface ou à la périphérie des phénomènes, comme par exemple dans les postures et procédures de distinction.

Faut-il pour autant renoncer à interroger les rapports sociaux dans leur pouvoir déterminant sur les réalisations humaines? Toute société sanctionne de façon plus ou moins codée des structures hiérarchiques et des formes ou forces de domination; toute société divise et classifie la masse confuse qui la compose; bref, toute société pratique le classement social et instaure (sinon institue) des rapports entre les classes qu'elle établit.

Peut-être ce concept déclassé retrouve-t-il quelque chose de son acuité et de son actualité lorsqu'on tente, comme nous nous proposons de le faire, de l'introduire dans l'explication des faits littéraires. Tentative à la fois simple et lourde d'implications que nous nous efforcerons d'ébaucher ici à la faveur d'une proposition volontairement limitée, comme de biais. Il va s'agir de montrer comment et dans quelle mesure la notion opératoire de classe peut s'appliquer non à des populations d'auteurs ou de lecteurs mais bien à des "populations" de textes. Ainsi procède-t-on spontanément lorsqu'on parle de "roman mondain» ou de "poésie populaire» et lorsqu'on distingue trois niveaux de la production culturelle : le haut, le moyen et le bas; le cultivé, le semi-cultivé et le trivial. Mais la sociologie littéraire n’a jamais généralisé ni même théorisé le concept de "classes textuelles". Nous voudrions poser ici les fondements de cette théorisation et, tout au moins, tracer ses premières perspectives.

\section{Textes et classes}

D'inspiration marxiste, la sociologie de la littérature a très tôt réservé à la notion de classe sociale un rôle éminent dans ses modèles explicatifs, son objet premier étant en l'occurrence de définir, pour une époque donnée, les relations nouées entre tel groupe social et tel groupe d'auteurs ou d'œuvres, entre telle classe et tel genre littéraire. Dans cette perspective, il s'agissait essentiellement de ratifier l'hypothèse selon laquelle tels produits textuels 
exprimeraient, dans leurs contenus comme dans leurs structures formelles, l'être collectif et l'idéologie du groupe social dont ils émanent. Suivant ce principe, Lucien Goldmann ou Erich Koehler ont sans conteste mis au jour des homologies convaincantes et contribué à donner, par la rigueur et les succès de leur démarche, une première légitimité à l'orientation sociologique des études littéraires. Tout autant, leurs travaux ont laissé entrevoir, malgré eux, que le postulat d'une homologie textes-classes ne pouvait rendre compte de toutes les situations et, en particulier, qu'il s'avérait des plus problématiques de le vérifier à chaque fois pour les productions esthétiques de l'époque moderne. C'est que, dans le même temps où le corps social gagne en mobilité et en complexité, la sphère des lettres affirme, en régime bourgeois et en économie de marché, une autonomie inédite dont l'enjeu et l'effet essentiels consistent à médiatiser son lien avec le champ global. Aussi les théoriciens des homologies ont-ils dû convenir de ce que la belle répartition méthodique dont ils rêvaient - distribuer et discriminer les œuvres selon les classes n'avait plus guère de pertinence ni de répondant concret au sein du système social des $X I X^{c}$ et $X X^{e}$ siècles. Deux voies de traverse, divergentes, se sont alors ouvertes à eux pour sortir de cette impasse: l'une les portait à renvoyer les auteurs et les cuvres à un vaste ordre bourgeois (ou petit-bourgeois) que la littérature ne pourrait pas ne pas reproduire ou contester; l'autre à disposer entre le littéraire et le social l'écran d'un jeu complexe de médiations. Soit par excès, soit par défaut, la référence au social tendait dans un cas comme dans l'autre à se dissoudre, et l'on vit mal ce qui subsistait désormais de l'ambition initiale.

\section{Textes et institution}

Plus récente, la théorie des champs symboliques ${ }^{2}$ ou de l'institution littéraire ${ }^{3}$ pose en d'autres termes et sur d'autres bases la question de la détermination du social sur les produits esthétiques. Pour elle la création moderne et ses agents ont pour site d'activité un espace spécifique, enclavé dans la totalité sociale et doté d'une relative autonomie de fonctionnement. Ainsi instituée, la littérature devient l'affaire d'une caste s'au to-gérant et vivant partiellement en vase clos. Indépendance toute relative, qui est le bénéfice symbolique retiré par l'écrivain de l'expansion du marché de la librairie lui permettant de se soustraire à ses anciennes tutelles. La théorie institutionnelle ne peut donc plus croire à une simple homologie entre classes, auteurs et œuvres (l'instance du littéraire et l'appareil qui le gère font écran entre les termes du rapport). Rejette-t-elle pour autant les divisions et distinctions

2. Voir les travaux de Pierre Bourdieu, notamment "Le marché des biens symboliques", dans L'Année sociologique, XX11, 1972, p. 43-126.

3. Voir Jacques Dubois, L'Institution de la littérature. Paris-Bruxelles, Nathan-Labor,. coll. "Dossiers Média ", 1978. 
sociales hors du champ de ses investigations? Certes non : elle aspire, tout au contraire, à les intégrer en ses modèles explicatifs de manière plus dialectique, en dégageant les rapports complexes et souvent ambigus qui s'établissent, à l'époque moderne, entre la sphère sociale et la sphère littéraire.

La période qui voit la littérature s'instaurer en champ clos et donner à ses pratiques une forme instituée est aussi celle durant laquelle la société (française par exemple) connaît une sécession forte entre ses composants, sécession activée et marquée par des antagonismes violents qui radicalisent les idéologies de classes. Mais le lieu d'ancrage du champ littéraire n'est guère la sphère générale des conflits; son horizon et ses bordures épousent les contours de la sphère bourgeoise et l'instaurent en espace indécidable, tout à la fois contenu en celle-ci et la contenant (la littérature relève de l'ordre bourgeois et l'excède). Composite et intégrant des couches nouvelles, animée par un principe de progrès et de mobilité, la bourgeoisie du XIX siècle et d'après lutte pour étendre ses pouvoirs et son champ d'action. De ce fait, elle s'étage en strates de plus en plus nombreuses, entre lesquelles les possibilités de circulation sont réelles : les études et le mariage, les carrières et l'argent ouvrent à ses tenants un carrefour de voies multiples, qui autorisent des changements de position. Sans doute cette mobilité, que célèbre le credo libéral et que décrivent les romanciers du temps, est-elle entravée par les forces d'inertie qui régulent la reproduction des grands corps; mais il n'empêche qu'au fil du temps, des bourgeois de plus en plus nombreux deviennent, par héritage familial ou par le jeu des carrières, des hybrides sociaux, composant avec plusieurs fractions de leur classe voire avec une ou des classes voisines.

Dans sa nouvelle définition, la sphère des lettres constitue l'un des accélérateurs de cette mobilité et l'un des creusets de ces hybridations. Par vocation elle est un domaine d'activité où la reproduction endogène est faible, où les fils ne succèdent guère aux pères et où toute émergence, tout positionnement nouveau résultent d'une promotion ou d'un déclassement. Parvenus et ratés, arrivistes et marginaux peuplent ce domaine et s'y croisent allègrement. Et cela d'autant plus que la condition d'écrivain ne bénéficie guère que d'un statut professionnel indécis et incertain : bien des auteurs composent avec un second métier, relèvent d'une autre filière socioprofessionnelle. Le journaliste émancipé côtoie le juriste en rupture, le petit fonctionnaire fréquente l'académicien. Ainsi, plus que tout autre corporation, la littérature s'avère perméable aux luttes et tensions sociales comme aux compromis idéologiques, mais elle ne l'est que sur un mode détourné. C'est qu'elle érige des concurrences initialement collectives en classements distinctifs des personnes et des personnalités. L'écho du conflit des classes ne s'y répercute, très étouffé, qu'entre ses multiples réseaux de relations interindividuelles.

Au principe d'un tel détournement intervient l'effet d'autonomie, mais d'une façon ici encore ambiguë. Si, d'une part, l'autonomie favorise l'hybridation sociale, elle tend de l'autre à la contenir voire à la neutraliser. Ainsi opère toute institution, dans la mesure où elle vise à homogénéiser le corps 
de ses agents. Mais la littéraire y procède par une manière de coup de force. Fondée sur un appareil peu structuré et dépourvue de tout code explicite (aucune juridiction ne la régente), elle engendre une idéologie intense qui est sans doute liée à sa fonction symbolique mais qui traduit surtout sa position marginale au sein du système global. Aussi transcende-t-elle la logique sociale qui la sous-tend en logique spécifique, jusqu'à instaurer un régime de rivalités et de classements qui apparaît, en dernière instance, comme une vaste métaphore du régime conflictuel général. Comme on l'a dit souvent, l'écrivain appartient à une fraction dominée de la classe dominante. Mais fort de la cléricature dont il relève et de la mission dont il s'investit, il s'autorise à retourner en positif son statut anomique, retraduisant celui-ci tantôt en appartenance objective à une communauté d'élus, tantôt en situation de distance subjective et subversive quant à l'ordre établi. En chaque cas, il feint d'échapper, comme Sartre l'avait bien vu, à l'emprise des déterminismes sociaux et des rapports de classes.

\section{La production des différences}

Dans le champ littéraire moderne, ces déterminismes sont si bien euphémisés et occultés qu'ils sembleraient privés de toute substance. Plus que d'y participer, les rivalités d'écoles et d'auteurs donnent l'impression de parodier les antagonismes de classes, de les mimer sur le mode du jeu et de l'artifice. Mais l'impression ne vaut que pour la scène étroite où se livrent les joutes les plus institutionnelles; elle s'estompe et s'évanouit dès que l'on prend en compte non plus la fine pointe de l'activité littéraire mais celle-ci dans son ensemble, en tant qu'elle instaure un vaste système où production et consommation ont partie liée. Sous cet angle, on voit revenir - massivement - une dimension sociale qui semblait perdue, provenant du pôle réputé passif de ce système, à savoir le public et le lectorat (destinataires dans le procès de la communication, consommateurs dans le rapport économique). Plus que par le passé - en raison alors de la faible diversité sociale du lectorat potentiel-a, la littérature se stratifie, à partir du $X I X^{c}$ siècle, selon des publics très différenciés. Hors champ, ces publics se distribuent sur une échelle de niveaux culturels et réintroduisent, à leur insu, les signes du social dans l'ordre autonome du littéraire. Comme par un effet d'équilibrage, la désocialisation des producteurs - corrélative de l'institutionnalisation de leur pratique - se trouve compensée par la diversifcation sociale des consommateurs, qui affectent de leurs marques de classes les textes dont ils passent, tacitement, commande. Cette stratification du champ des textes par l'instance lectorale résulte en effet de l'inscription de la production littéraire dans une économie de marché, qui la contraint à couvrir l'ensemble du public potentiel et dès lors à rencontrer la demande particulière de chacune de ses fractions. A chaque couche de lecteurs, la lecture qui lui convient et les textes dans lesquels elle peut se reconnaître. C'est là une 
tendance forte de la littérature contemporaine que de classer ses productions (et de produire des produits classés) en référence aux classements - effectifs, supposés ou construits - des différents lectorats visés ${ }^{4}$.

Mais qui classe? En principe ce sont les producteurs ${ }^{5}$ qui hiérarchisent les produits en fonction des consommateurs. Il serait cependant fallacieux de supposer chez eux une parfaite conscience et une entière maîtrise de leurs manœurres classificatoires. La démarche en cause - et la "politique " qui lui est implicite - s'avère prise dans une logique circulaire qui veut que quiconque classe se classe lui-même, et réciproquement. Au principe d'un tel «tourniquet» il y a le fait fondamental, observé déjà par Marx, que l'objet produit le sujet - pas seulement celui de la consommation mais aussi, dans une certaine mesure, celui de la production même. L'auteur est l'enfant de son texte, l'éditeur celui de ses livres, textes et livres toujours déjà impliqués et définis dans le vaste système des modes et des modèles. La question de savoir "qui classe? " renvoie donc à un procès sans fin, et dont l'origine s'avère indéterminable.

Ainsi, les procédures de classement et la ou les grilles de classes textuelles installent, au cœur même de l'appareil littéraire, un régime discriminatoire et distinctif qui est le répondant symbolique du système des classes sociales et de leurs antagonismes. Mais ce régime est aussi spontanément reconnu dans son existence brute (chacun admet que le corpus appelé littérature est stratifé en textes plus ou moins élaborés et orientés vers des lectorats distincts) que méconnu dans sa portée et sa signification sociales (seuls sont admis le critère sélectif de la qualité et l'argument mercantile du public ciblé). De là que l'incidence des effets sociaux sur les catégories de textes n'ait été abordé que de façon occasionnelle. Fait d'autant plus étonnant que la recherche moderne, en retravaillant la notion mieux accréditée de genre, a exploré un domaine mitoyen où ces effets, comme nous allons tenter de le montrer, sont non moins actifs.

4. "La lutte des classements, écrit Pierre Bourdieu, est une dimension fondamentale de la lutte des classes. » Et il y va du pouvoir politique même puisque, selon l'auteur de Choses dites, classer c'est * imposer une vision des divisions" et donc exhiber son "pouvoir de faire des groupes, de manipuler la structure objective de la société » (Espace social et pouvoir symbolique ", Choses dites, Paris, Minuit, coll. "Le Sens commun ", 1987, p. 64). Ainsi, il existe une "politique de la littérature" qui agit par et sur l'échelle des degrés culturels et joue d'un subtil dialogue entre production et consommation. Politique stratifiante déjà effective dans l'ancienne littérature - les trois styles de la poétique antique - mais qui atteint, dans la littérature moderne, un degré de complexité (et peut-être de duplicité) jusque-là inédit.

5. Entendons par là non seulement les écrivains mais tous ceux qui régissent, de l'intérieur ou de l'extéricur, l'appareil littéraire : éditeurs, critiques, professeurs. Les premiers déterminent notamment la collection dans laquelle sera présenté le produit; les deuxièmes sélectionnent, dans l'ensemble des produits, ceux qu'ils estiment destinés au public qu'ils représentent (dans les différents médias); les troisièmes sanctionnent le "tri de la postérité " et stratifient le corpus littéraire selon les degrés et le réseau de leur enseignement et selon leur représentation personnelle de ce corpus. 


\section{L'instance générique}

En littérature les genres représentent la grande forme classificatoire subsumant tous les autres modes de catégorisation. Dès l'origine ils ont forme instituée, en tant qu'ils composent l'unique code prescriptif explicitement établi, et force instituante, en ce qu'ils donnent à connaître et reconnaître ce qui est littéraire et ce qui ne l'est pas. La notion de littérature peut être sujette à caution; celles de poésie ou de tragédie ont un pouvoir d'évidence universelle qui lève tous les doutes. Données élémentaires de la perception et de l'expérience, formes régulatrices du système des lettres, les genres n'en occupent pas moins les uns envers les autres des positions variables. D'une époque à l'autre, ils peuvent changer de valeur, de portée, d'aspect. Aussi peut-on jusqu'à un certain point leur attribuer la fonction historique reconnue par Roland Barthes aux "écritures" 6 , à savoir qu'ils témoignent, comme elles, d'un engagement particulier de l'écrivain, face à la société et face à l'institution littéraire. Opter pour la poésie en France a des résonances très diverses selon que l'on pose ce choix en 1820, en 1870 ou en 1920. Élire en 1980 le sonnet plutôt qu'une forme libre constitue en soi un acte "politique" autant qu'esthétique. Bref, comme les "écritures", les genres trahissent ou exhibent un positionnement (pose adoptée, distance fixée, mode prévu d'intervention, etc.)

Régissant du plus haut l'espace des formes, les genres l'animent d'un double principe de liberté (celle de choisir) et de contrainte (celle de devoir choisir et de ne le pouvoir qu'entre quelques possibles). Mais s'ils sont, comme on l'a dit, des codes essentiellement prescriptifs, il n'en reste pas moins que, d'époque en époque et d'esthétique en esthétique, leurs prescriptions varient de contenu et de degré d'exigence. Somme toute, la seule grande loi qui se maintienne est celle, essentiellement proscriptive, qui prohibe le mélange des genres. Nécessité d'un ordre, tabou frappant toute confusion.

Leur prééminence et leur surdétermination sont telles que les grands genres tendent à former des institutions dans l'institution, selon une sorte de balkanisation du système ${ }^{7}$. Le roman, la poésie et le théâtre font enclave dans le champ littéraire; leurs agents et leurs productions relèvent d'"univers séparés ${ }^{8}$ dont chacun instaure et gère son propre code (d'écriture, de poses, de postures) mais qui n'en sont pas moins en situation de concurrence : ainsi du match poésie/roman, gagné par les romanciers au terme du XIX ${ }^{c}$ siècle et relancé (en vain) par les surréalistes. Genre proliférant, vorace, annexionniste, le roman n'a dépossédé poésie et théâtre de leurs privilèges exclusifs qu'afin

6. Roland Barthes, Le Degré zéro de l'écriture, Paris, Seuil, coll. "Points ", 1972.

7. Au point d'ailleurs qu'ils finissent par désigner des faits extra-littéraires: un paysage "poétique", une "tragédie " de la route, une "épopée " sportive...

8. Ainsi on dit plus volontiers "romancier " ou "poète" qu' écrivain ", désignations particularisantes qui attestent sans doute la prégnance de notre représentation générique de la littérature mais qui nous paraissent de surcrồt - el peut-être davantage - résulter d'un transfert, dans l'ordre littéraire, du principe bourgeois de la division du travail. 
d'exprimer sa primauté dans la conquête d'immenses territoires, et non pour conquérir un haut prestige. L'essai lui fait bonne escorte. Durant quoi, le théâtre et la poésie se voient relégués dans des positions minoritaires qui les instaurent en "univers séparés", ceints de l'aura désuète d'un capital peutêtre révolu et figurant en abyme la clôture du système global.

Outre qu'elle garantit la littérarité, l'instance générique contribue à la socialisation symbolique et/ou institutionnelle des textes et des auteurs qui observent ses injonctions, en les inscrivant dans une population d'auteurs et de textes apparentés. Opter pour tel genre, c'est en effet, si l'on y regarde bien, faire allégeance à un patrimoine en greffant sa production dans le grand corps intertextuel des productions répondant au même canon et payer de sa fidélité au modèle la caution symbolique qu'il confère au produit et à son producteur. De là que le système moderne (en son pôle traditionnel) privilégie la spécialisation générique et ne puisse tenir Victor Hugo, homme-orchestre, que pour un monstre polymorphe, à la fois institution à lui seul et contreinstitution, " confisqu[ant] chez qui pense, discourt ou narre, presque le droit à s'énoncer ${ }^{9} "$.

\section{Les genres dans l'institution}

Selon leur effet institutionnel majeur, qui conjugue autorité et prestige, stabilité et ségrégation, les genres se donnent pour origine et pour système (ils ont force de loi, paraissent toujours antérieurs à tout texte). Chaque fois qu'elle s'est appliquée à ontologiser l'ordre générique - et de pétitions de principe en «bricolages " 10 , elle n'y a pas manqué -, la poétique n'a fait qu'abonder en ce sens, soucieuse de dégager sous la concrétion des produits littéraires apparentés la ou les grandes catégories essentielles dont ccux-ci procéderaient. On commence à voir que ce point de vue, pour être très reçu, inverse dans une large mesure l'ordre réel des choses. Outre qu'elle néglige le fait que maintes formes génériques ont un statut historiquement défini, la conception systémique laisse entrevoir qu'elle dépend d'une construction a posteriori. Ainsi et tout d'abord, l'appareil générique dont notre époque hérite n'est que trop visiblement un agrégat de formes renvoyant à des strates très diverses. Par ailleurs, même dans les cas où il s'avère possible d'accorder à certains genres un fondement anthropologique - la fonction narrative, par exemple -, leur définition repose sur la cristallisation d'une conjoncture littéraire autour de quelques textes de référence. En somme, les textes produisent les genres plus qu'ils ne les reproduisent (l'objet semblant en quelque sorte

9. Mallarmé, "Crise de vers *, dans CEuvres complètes, Paris, Gallimard, Bibliothèque de la Pléiade, 1945, p. 360-361.

10. Le mot, pour désigner les constructions systématiques des poéticiens, est de Gérard Genette, dans son Introduction à l'architexte, republié dans l'ouvrage collectif Théories du genre, Paris, Seuil, coll. "Points ", 1986. 
contenir la catégorie dont il relève). C'est à quoi, dans une perspective différente de la nôtre, Jean-Louis Schaeffer rend attentif lorsqu'il écrit:

"Si nous en restons au niveau de la phénoménalité empirique, la théorie générique est tout simplement censée rendre compte d'un ensemble de ressemblances textuelles, formelles et surtout thématiques: or, ces ressemblances peuvent parfaitement être expliquées en définissant la généricité comme un ensemble de réinvestissements (plus ou moins transformateurs) de cette même composante textuelle. La littérature étant par définition institutionnelle, la généricité peut parfaitement êtrc expliquée par un jeu de répétitions, d'imitations, d'emprunts, etc., d'un texte par rapport à un autre, ou à d'autres, et le recours à un postulat aussi " puissant " que celui d'une structure ou matrice de compétence se révèle parfaitement superflu puisqu'il n'explique pas plus de choses que ne le fait une conception transtextuclle de la généricité. En plus ce postulat est inadéquat, dans la mesure où il est incapable de prendre en compte la dimension essentiellement dynamique de la généricité et impose une vue simplement classificatoire qui méconnaît la spécificité de la relation générique "."

L'analyse de Schaeffer introduit à la notion de classes de textes, sans pour autant lui reconnaître une portée sociale. A ce stade, dire que les textes se distribuent en classes revient à soutenir qu'ils forment des groupes perçus comme catégoriels et que, pour un temps, le groupe "classé "va faire office de référence et de modèle pour d'autres textes, avant de se défaire ou de se fractionner en sous-groupes susceptibles de s'autonomiser. Évoquant ce double procès, d'instauration en référence et de dégénérescence, Schaeffer avance l'hypothèse ingénieuse selon quoi l'agénéricité revendiquée par les tenants de la modernité ne serait jamais que l'avers d'une activité générique proliférante:

"Le développement de la circulation littéraire (dû à des causes technologiques autant que sociales) au cours des derniers siècles a comme conséquence une multiplication extrême des modèles génériques potentiels, en sorte que l'activité générique (liée à la réflexivité de plus en plus prononcée de la littérature dite "sérieuse") très poussée des textes modernes aboutit à une telle multiplication générique que les classifications sont très difficiles à établir ${ }^{12}$."

Mais c'est par un aspect plus incident que la réflexion de Schaeffer retient surtout notre attention. Lorsque, en rupture avec la doctrine ontologique dominante, il s'astreint à un point de vue strictement phénoménologique allant du texte au genre-, l'analyste donne à voir le travail de l'instituant sous l'institué. A l'institué correspond le pouvoir de normativité et de régularité systémique qui nous a porté à soutenir que les genres valaient comme institution(s); à l'instituant le travail de fondation et de transformation exercé par les textes sur les genres, travail des plus intenses dans l'ère moderne. Ainsi,

11. J.-M. Schaeffer, * Du texte au genre. Notes sur la problématique générique », dans Théories du genre, éd. cit., p. 186.

12. Ibid., p. 202. 
au sein d'un champ en mouvement, le genre est non seulement classe, mais classe en train de se faire et de se défaire. Double logique de l'identité et de la différence, mieux: de l'identité dans la différence : car le genre est aussi cela qui ne se maintient qu'en se transformant, mobilité paradoxale évoquant quelque peu celle des structures de la bourgeoisie.

\section{Genres ou classes, genres et classes}

L'ordre qui préside à l'orientation lectorale et à la distribution catégorielle des textes constitue bien plus, on s'en doute, qu'un préalable obligé, assez inoffensif : genres et classes sont tout à la fois, dans ce champ de forces qu'est la littérature, les enjeux et les armes d'incessantes confrontations; par eux, les acteurs conquièrent leurs titres et, grâce à eux, leur rang dans l'institution. Nous en venons ainsi à la question centrale : si les textes participent de classes génériques, ces classes ont-elles une dimension sociale? Autrement dit, les genres sont-ils des classes ou entretiennent-ils des rapports avec celles-ci?

On postulera qu'il existe toujours une relation entre genres et classes, même si cette dernière n'a rien d'univoque ni de constant. Historiquement, cette relation paraît s'exprimer selon deux formes d'alliances distinctes et concurrentes. La première tend vers la superposition des genres et des classes, par quoi coïncident leurs découpages respectifs, et laisse supposer qu'une hiérarchie spécifiquement sociale est rabattue sur une hiérarchie générique. Genre noble aux époques classique et romantique, la poésie jouit d'une légitimité que lui confèrent ceux qui la pratiquent (nobles ou grands notables); après quoi, pratiquée par la génération des petits fonctionnaires - tels Verlaine ou Mallarmé -, elle doit compenser son déclin social par un discours d'escorte mythifiant et préserver sa légitimité compromise par une opacification luxueuse de son langage. La seconde forme d'alliance entre genres et classes se présente comme une intersection "verticale» entre les deux échelles. En ce cas, les genres sont stratifiés en classes hiérarchiques, ce qui signifie aussi bien que les classes se subdivisent en genres. A côté de la poésie haute peuvent exister une poésie moyenne ou bourgeoise et une poésie basse ou populaire. Au cours des temps, les deux régimes ont dû plus d'une fois se faire concurrence, s'ajustant l'un à l'autre vaille que vaille.

On est tenté cependant de penser que le régime de la coïncidence répond à un état traditionnel du champ littéraire (état où prime la force de l'institué) cependant que la stratification des genres en classes s'accorde à la littérature de marché, cette littérature moderne qui est encline à la prolifération générique dissolvante. La seconde moitié du XIX ${ }^{e}$ siècle est, de ce point de vue, intéressante à étudier, en ceci que les deux régimes entrent en collision jusqu'à susciter des distorsions flagrantes. Ainsi la poésie du Parnasse, voire celle du symbolisme, peut encore se croire reine légitime selon le premier régime, alors que le roman déjà a gagné la partie en s'alignant sur le second régime, qui l'induit 
à se diversifier en modalités variées et socialement finalisées (du "roman populaire " au "roman expérimental » ou " psychologique "). Fait de démocratie littéraire - le roman "pour tous"-, une telle victoire s'accompagne donc d'un dispositif compensatoire - une classe de romans pour chaque classe sociale -, dont on pressent combien il est déterminé par les lois du marché ${ }^{13}$.

Qu'il s'agisse du premier ou du second régime, jusqu'où les dispositifs hiérarchiques sont-ils avoués, affichés, admis et reconnus? Toute une enquête devrait être menée sur ce point, tenant compte des pratiques d'époque et de la variabilité des échelles de la légitimité. En se cantonnant une fois encore aux deux derniers siècles, il est permis de dégager quelques tendances. En règle générale, le genre ne s'indexe pas explicitement sur sa ou la classe. Les exceptions - "roman populaire » ou "roman mondain » - ont un air de candeur et s'avèrent de toute façon ambiguës, comme on l'a plus d'une fois relevé (romans par, pour ou sur le peuple/les mondains?). Pour peu qu'il soit socialement marqué (et, en l'occurrence, démarqué), le genre préfère même se dérober. La consonance triviale du mot «roman» a voulu, jusque tard dans le $\mathrm{X} I \mathrm{X}^{\mathrm{c}}$ siècle et alors même que le genre romanesque triomphait, que la dénomination soit omise sur la couverture d'une grande partie des œuvres publiées. Est-ce à dire que le genre efface méthodiquement ses références sociales? Plus exactement il montre en cachant. Fonctionnant sur le mode de l'indice, il est toujours plus ou moins désignation métonymique de sa classe et de ses propriétés. Quelle que soit sa position, l'usager ne s'y méprend guère, se reconnaissant en tout cas dans les genres qui sont faits pour lui et qui, de manière oblique, le pointent.

Soit, à titre d'exemples, quatre appellations actuelles, distribuables sur une même échelle: 1) "roman d'amour et d'aventures"; 2) "série noire"; 3) « roman»; 4) « récit». La plus neutre - «roman» - correspond, semblet-il, à la production moyenne (traditionnelle) pour public moyen (quelconque). De part et d'autre, la nomination dévie : la littérature d'avant-garde essentialise et euphémise - "récit " - tandis que la production triviale ou semi-triviale en rajoute, notamment par spécification plus ou moins métaphorique des contenus - "série noire", "romans d'amour et d'aventures". Pour être inverses, les deux tendances extrêmes figurent la même opération : diversifier et spécifier pour affiner le classement (à chacun son genre) mais tout autant pour raffiner le code même du classement. Ni les modulations distinguées sur la désignation en litote ni la surdétermination triviale à valeur hyperbolique ne trompent: chacun sait de quel côté il se trouve et chacun retrouve, avec bonheur, un code qui, quoique allusif et parce que allusif, dit bien ce qu'il dit.

13. Dans Production de l'intérêt romanesque (La Haye, Mouton, 1973, p. 52), Charles Grivel a donné une première description, pour la seconde moitié du XIX siècle, de l'édifice moderne dus roman, disposé en quatre étages: réaliste ou naturaliste, bourgeois ou romanesque, honnête. populaire. 


\section{De la hiérarchie au feuilletage}

Ainsi, sous le (pseudo-)système des genres, se tient un système de classes textuelles plus organique et plus déterminé. Comment le décrire? Loin d'être une structure close ou figée, il s'enrichit et se remodèle au gré des conjonctures. De plus, par-delà la vaste tripartition en sphères de production/consommation sur laquelle il repose, on découvre un feuilletage, potentiellement illimité, en strates découpant les sous-classes. Comment objectiver une telle échelle distributive? Comment en fixer les niveaux, ne serait-ce que pour un état donné du champ? Deux types de critères - externes et internes - permettent d'esquisser une première modélisation. Le recours aux critères externes consisterait à isoler ces strates en fonction des groupes lecteurs, de leurs choix, de leurs usages ${ }^{14}$. Problématique à conduire pour le passé, l'enquête prend toute sa portée pour le présent. Elle risque cependant d'achopper sur la difficulté de conjuguer l'homogénéité des classes textuelles avec celle des groupes qui les reçoivent et qui peuvent quelquefois n'être pas ceux visés par ces classes. Les exemples ne manquent pas de tels décalages ou ricochets, depuis la poésic hermétique, à vocation élective, goûtée par un lectorat venu des couches moyennes (qui sanctionne sa promotion sociale par des lectures hautement distinctives) jusqu'au roman policier qui fait les délices de la classe intellectuelle (encanaillement dans un genre populaire, mais aussi usage ludique d'une forme narrative à transformations réglées). Le recours aux critères internes revient, quant à lui, à repérer les indicateurs sémiologiques dessinant la configuration d'un classement, opérant tant sur le registre littéraire que sur le registre socioculturel is. Ici le risque est grand de devoir travailler sur des paramc̀tres aussi ténus que foisonnants, et parfois impondérables.

Toutefois, si ce type d'enquête ou d'inventaire peut incontestablement fournir à la recherche des formes d'étalonnage, la théorie des classes textuelles doit surtout tenir compte de ce que celles-ci ne sont pleinement reconnaissables et définissables qu'à la rencontre de certains rapports et de certains effets.

14. On trouvera un exemple accompli d'une telle démarche dans l'article de Patrick Parmentier, "Bon ou mauvais gense. La classification des lectures et le classement des lecteurs , in Bulletin des Bibliothèques de France, t. 31, $\mathrm{n}^{\circ} 3,1986$, p. 202-223.

is. La destination sociale des produits esthétiques se laisse ainsi entrevoir dans des séries d'éléments récurrents, au couur et surtout à la périphérie des textes, qui fonctionnent, en définitive, comme autant d'indicateurs sémiologiques de classements. Au " seuil " du livre, le producteur (et singulièrement l'éditeur) dispose ainsi un réseau de marques qu'il contrôle plus ou moins bien mais qui finissent tôt ou tard par excéder son contrôle, jusqu'à prendre en certains cas un autre sens que celui dont elles étaient initialement investies. Dans Seuils (Paris, Éd. de Minuit, coll. "Poétique*, 1987), Gérard Genette a fait un relevé cadastral et un inventaire très détaillés de ce domaine du paratexte - éditorial et auctorial - en quoi se disposent les indicateurs en question. Du point de vue de leur effel classificatoire, les indices les plus efficaces sont la présentation (figurative ou non) de la jaquette (présente ou absente), le titre, l'éventuelle dénomination générique, les noms de l'éditcur et de la collection, le prière d'insérer. Ils constituent un code sommaire, d'une incontestable efficience dans le commerce de la librairie (le livre se donne dabord comme objet). On peut regretter que Genette, retranché derrière la perspective strictement poéticienne de sa recherche, ait méconnu l'investissement social de ces marques para-textuelles, sous le prétexte (à notre sens fallacieux) que cet investissement s'avère difficilement théorisable. 
Entités évanescentes qui s'évanouissent dès que les sujets classés et classants (lecteurs, éditeurs, auteurs) cessent d'y être sensibles, elles relèvent avant tout de l'ordre des représentations. C'est en partie, aujourd'hui, le rôle de l'appareil producteur d'en tracer les contours (sans trop les accuser) et de les remodeler selon l'évolution possible du marché telle que le marketing du livre permet de la pressentir.

Pour ce qui concerne les "rapports", ils sont, dans le jeu littéraire comme dans le jeu social, plus intimement fondateurs, puisque toute classe se définit dialectiquement selon sa position quant aux autres. Mieux: il n'est pas de classement qui échappe à un procès de définition réciproque et d'ajustement mutuel. Nous sommes ici au cœur de la théorie de la distinction (Bourdieu), selon laquelle quiconque classe se classe en classant. Toute la stratégie de nos options et de nos préférences nous tourne en effet vers des objets ou des pratiques déjà définis socialement et qui nous renvoient inlassablement à notre appartenance, en sorte que nos sélections confirment et confortent celle-ci. Mais le mouvement, comme on l'a déjà suggéré plus haut, est circulaire et les objets choisis sont en retour renforcés dans leur définition sociale. Pour saisir au mieux l'importance de cette interaction, on peut invoquer la relation qu'entretiennent éditeurs et lecteurs. Tel éditeur confère à ses produits certaines marques génériques (selon les modalités que nous avons indiquées) de manière à atteindre un certain groupe de lecteurs; il opère donc un choix, dont il exhibe les emblèmes, mais en fonction d'un public qui, à ce moment, reste encore largement virtuel, voire fictif. Classant des produits et leurs consommateurs espérés, l'éditeur se classe: ajoutons d'ailleurs qu'il ne classe les produits qu'à partir de l'idée qu'il se fait de son propre classement. Après quoi intervient le public réel ou contingent, qui va reproduire l'opération en sens inverse. Socialement défini, le groupe consommateur reconduit, par ses choix, la position de l'éditeur au sein des hiérarchies sociosymboliques et, ce faisant, définit plus finement sa propre position. Quel que soit son sens, l'effet de classement - on l'aura compris - tient à la fois de la reproduction et de l'ajustement. D'un côté, je reflète et reproduis mon statut social par le jeu de mes options; de l'autre et dans un perpétuel mouvement de mise au point, je projette dans mes options une idée de moi retouchant la réalité que je suis. De là que la circularité du procès classificatoire ne l'enferme pas dans la sphère d'une répétition figeante.

\section{Hétéro/autonomie des genres ou classes de textes}

Si les classements littéraires résistent à l'appréhension théoricienne, c'est sans doute parce qu'ils proviennent d'une interaction complexe entre instances de production et instances de consommation; mais c'est aussi parce qu'ils se produisent ou se reproduisent au croisement de deux grands ordres de détermination - l'un symbolique, l'autre social - et des deux logiques distinctes 
propres à celles-ci, qui tantôt se redoublent et tantôt rivalisent. Ici interviennent des mécanismes mettant en jeu aussi bien l'exigence économique la plus élémentaire (rentabiliser, par exemple, le lancement d'un genre particulier comme le roman peplum) que la distinction symbolique la plus subtile (fournir à un groupe restreint d'intellectuels ses emblèmes textuels). Ces mécanismes correspondent à un chapitre de la sociologie littéraire qu'il est d'autant plus urgent d'ouvrir que, comme le note Vincent Descombes dans son récent ouvrage sur Proust, la hiérarchie des valeurs et le classement par rangs relèvent de l'esprit spécifique des institutions (françaises), celui même que l'école inculque très tôt aux sujets sociaux ${ }^{16}$ et qu'en l'occurrence, La Recherche du temps perdu ne cesse de dévoiler ${ }^{17}$.

On se gardera cependant de limiter l'instauration des classes textuelles à la seule action réciproque du champ littéraire et des fractions de la société globale - les lectorats - qui sont orientés vers lui et vers qui il s'oriente. Il importe en effet de tenir compte de la capacité de ce champ à exercer sa logique. L'incessant modelage des genres et les variations de leur distribution hiérarchique sont partiellement le fait d'un dispositif régulateur purement institutionnel. Autrement dit, les facteurs internes médiatisent de façon variable, selon les époques et les conjonctures historiques, l'effet des facteurs externes. Dès le romantisme, les genres sont pris dans la lutte concurrentielle qui oppose les écrivains en quête de la gloire et du pouvoir symboliques; à partir du Parnasse et de l'école réaliste, les programmes esthétiques rivaux vont jouer un genre contre un autre, voire faire jouer un genre contre lui-même (par surou sous-codage de ses marques). Ainsi, de Leconte de Lisle au surréalisme, de Zola à Robbe-Grillet, le duel entre poésie et roman nourrit un siècle de controverses, auxquelles prennent part, en retrait, des genres esthétiquement dérivés et des formes mineures comme le théâtre avant-gardiste (symboliste et post-existentialiste) ou l'essai (de Michelet à Barthes).

\section{Genres de comportements}

Mais, pour ceux qui sacrifient à leur loi, les genres ne sont pas que des instruments stratégiques, ni de simples médias, supports contingents d'un message seul essentiel. En effet, si l'appareil générique constitue, comme on l'a vu, une instance socialisatrice garantissant à l'écrivain son statut et à ses produits leur littérarité, on peut observer que chaque genre tend non seulement à imposer aux textes des structures thématiques ou formelles mais encore à dicter aux auteurs qui s'y réfèrent un code de poses, des règles de vie: en

16. V. Descombes, Proust, Philosophie du roman, Paris, Éd. de Minuit, 1987, notamment aux p. $135-136$.

17. * Car je continuais à leur demander avidement de classer les différents officiers dont je savais les noms, selon l'admiration plus ou moins grande qu'ils leur semblaient mériter, comme jadis au collège, je faisais faire à mes camarades pour les acteurs du Théâtre-français. "(Proust, Le Côté de Guermantes, I, Paris, Garnier-Flammarion, 1987, p. 202). 
somme, un mode d'être. Ainsi les symbolistes, Mallarmé en tête, ont-ils transféré dans le rituel de leurs réunions et dans certains comportements calculés les principes de leur credo générique. Tendance ouvertement manjfestée et radicalisée par les surréalistes lorsque, soucieux de restituer au genre poétique son aura, ils l'hypostasieront en principe de vie incitant à l'action révolutionnaire. Ceci dit, on peut se demander si de telles objectivations existentielles procèdent d'une identification au genre élu ou du programme littéraire particulier dont on l'investit : le romancier "artiste " à la façon de Barbey d'Aurevilly doit sans doute moins au genre qu'il pratique qu'à son credo esthétique, son dandysme réactionnaire et ses postures désinvoltes. II reste cependant qu'un genre à forte codification tel que la poésie suscite tendanciellement chez ses pratiquants, du romantisme au surréalisme et en dépit des variations programmatiques des écoles successives, les mêmes attitudes et les dote d'une même conscience, quasi religieuse, de leur statut et de leur "mission" 18.

\section{Transferts et transactions}

La lutte des classements, corrélative de la concurrence des écoles, oppose sans doute des strates très localisées du champ textuel ou pré-textuel (les matrices génériques) mais elle engage aussi, à mieux y regarder, l'ensemble du système. Chaque catégorie ou classe se défnissant par rapport aux autres, toute transformation en un point de la structure littéraire est en principe susceptible de s'y diffuser de strate en strate, jusqu'à l'altérer dans sa totalité. Vue théorique, excessive, que ratifient cependant, de façon partielle, les séries d'intermutations enregistrées par l'analyse historique des classes textuelles. Ainsi, on observe qu'à l'occasion de ruptures novatrices, se met en branle un processus de régulation lui-même autorégulé qui garantit à deux égards la stabilité "économique" du système, en tant, d'une part, qu'il entrave l'emballement des réactions en chaîne et, d'autre part, qu'il procède aux redispositions réciproques indispensables. Trois exemples, de complexité croissante, le feront voir à l'œuvre. Le premier consiste dans le cas simple de deux sousgenres entrant en interaction dès le moment où l'un d'eux est pris dans un mouvement transformateur auquel l'autre réagit; le deuxième, déjà plus subtil, donne à voir un effet d'ajustement dialectique entre deux genres distincts; quant au troisième, il représente un cas complexe de «transaction " symbolique dans la mesure notamment où celle-ci s'opère non plus entre deux types de production littéraire, mais, plus largement, entre deux types de production discursive.

A. Les cinq dernières années du Second Empire voient la double appa-

18. Ajoutons que la représentation ordinaire du * poète * (comme personnage bohème, évanescent et délicat) confirme - serait-elle même fictive ou illusoire - cette appropriation comportementale du
sujet écrivain à son statut. 
rition du roman artiste des frères Goncourt et du roman judiciaire d'Émile Gaboriau. Fait de synchronie passé inaperçu, et cela d'autant mieux que les deux types romanesques relèvent de zones très distinctes de l'institution littéraire. D'un côté, avec Manette Salomon et Madame Gervaisais, les Goncourt engagent leur genre d'élection dans la voie, alors toute neuve, de la "modernité »: affaiblissement de l'intrigue, fragmentation du récit, point de vue oblique, effacement du sujet-héros. De l'autre, Gaboriau fait dériver du roman-feuilleton un type de fiction finalisée à l'extrême et tributaire d'une stricte logique narrative, cependant qu'un héros enquêteur vient occuper en permanence le devant de la scène et imposer à l'action sa forte autorité. En somme, ce à quoi renonce un sous-genre, un autre le reprend sous une forme insistante, hyperbolique. Il n'est pas indifférent que cette fonction supplétive et compensatoire soit assumée en l'occurrence par un "genre " neuf et appelé, parti du bas, à s'élever peu à peu dans l'échelle des valeurs. Le roman judiciaire vient ainsi, en quelque sorte, occuper un territoire abandonné, en même temps qu'il semble alerter, par l'excès de sa structuration narrative, la "conscience littéraire" quant aux risques que font encourir au système les renoncements de l'avant-garde. Quoi qu'il en soit, les deux formes concurrentes induisent un réaménagement du secteur romanesque. Le caractère orienté et transactionnel de leur relation mutuelle apparaît d'évidence lorsqu'on perçoit, derrière leurs violentes divergences, des options convergentes instaurant, entre les deux formules, un dialogue second. Ainsi on peut relever que le héros effacé des Goncourt est, à sa manière, un dandy décrypteur d'énigmes (à l'instar du policier Lecoq) et que la structure prégnante du récit judiciaire dissimule plus ou moins bien le caractère fragmenté et fragmentaire de l'histoire du crime (que partagent les narrations "artistes" des Goncourt) 19 .

B. Quelques années plus tard, dans les débuts de la Troisième République, un phénomène analogue de concurrence à fonction compensatoire se produit entre deux genres pour lors en pleine rivalité : d'un côté, le roman en voie de légitimation; de l'autre, la poésie dont s'amorce le déclin. Dans sa variante symboliste, la poésie fin-de-siècle, en conformité avec son credo d'une pratique purement langagière et autoréflexive, pose en des termes radicaux et négatifs la question du sujet de l'énonciation poétique : poussant à sa limite le précepte parnassien de l'impassibilité, Mallarmé et ses émules prônent et pratiquent une poésie "impersonnelle ", procédant à « la disparition élocutoire du poète " ${ }^{20}$. Ainsi le mouvement poétique dominant au terme du siècle rompt avec la représentation romantique (et littérairement codée) de l'activité lyrique comme miroir du moi ou journal intime des émotions subtiles. On ne peut qu'être frappé de ce que, dans le même temps, émerge une école nouvelle, conduite par Bourget et Barrès, dont les productions romanesques et l'idéologie thématisent et proclament la primauté absolue du sujet individuel, exaltent le

19. Voir Jacques Dubois, * Un cas de transfert symbolique, roman artiste et roman judiciaire à la fin du Second Empire ", dans CEuvres et critiques, XII, 2, 1987, p. 35-47.

20. Mallarmé, "Crise de vers ^, dans CEuvres complètes, ćd. cit., p. 366. 
"culte du moi » et consacrent la psychologie des passions comme modèle explicatif des comportements sociaux et comme savoir scientifique présidant à l'imagination narrative. Selon un mécanisme déjà actif dans le cas Goncourt/ Gaboriau, les "romanciers psychologues" fondent en somme la doxa de leur école sur les ruines d'un domaine déserté par les poètes et, comme dans ce cas, l'opérateur de la reprise est un genre en ascension. Occupation opportuniste d'un site abandonné, sans doute; mais aussi, ce qui n'était pas exactement le cas dans l'exemple précédent, reprise en charge à portée stratégique d'un "canon" traditionnel dont l'abandon par l'école rivale cause, chez un public dérouté, la condamnation de cette dernière (accusée de byzantinisme desséchant, de démence formaliste, d'esthétisme artificiel et glacé). On notera encore qu'en l'espèce, le modelage inversé d'un genre sur l'autre s'est probablement opéré par la médiation du roman symboliste, qui a transféré à l'ordre romanesque le précepte mallarméen de l'abolition du moi, y procédant, comme cela apparaît dans les fictions des Dujardin, Paul Adam, Camille Mauclair ou Remy de Gourmont, par un constant décentrement de l'instance subjective, émiettée, disséminée dans la dérive d'une conscience évanescente ${ }^{21}$. Aussi la manœuvre des romanciers psychologues fut-elle double: d'une part, entraver la percée d'un mouvement romanesque avant-gardiste, issu du symbolisme, et, d'autre part, s'imposer en exhibant, contre celui-ci, sa conformité à un canon littéraire établi, tout en fournissant au lectorat moyen des romans «vraisemblables" et valorisés par leur (prétendue) dimension éfique. Enjeux essentiellement stratégiques, et témoignant des rapports de concurrence et de domination qui se jouent derrière ou à l'origine des faits de redispositions réciproques des classes de textes.

C. Le dernier cas exemplaire que nous voudrions évoquer ici déborde les cadres stricts du champ littéraire tout en restant enclos dans celui de la production écrite. Il concerne les effets induits sur la sphère restreinte des lettres par l'expansion, après 1885 , du secteur médiatique et, corrélativement, par le développement accéléré de la production para- ou infra-littéraire. Étudiée par Marc Angenot ${ }^{22}$, l'extension de la grande presse - dont on débat beaucoup à l'époque dans les milieux littéraires - fait notamment craindre parmi ceuxci qu'elle vienne à entamer l'aura de la "chose écrite», en proposant au public une variante dégradée tant de la culture scripturale que de l'objet-livre luimême. Crainte partiellement fondée puisque, autour de 1890, la presse, qui est très demandeuse de textes divers (romans, contes, chroniques) souvent réunis en volume après parution quotidienne, va très vite engorger le marché par un flot d'ouvrages médiocres, banalisant le support prestigieux du livre, et provoquer ce que les contemporains ont nommé le "krach de la librairie". L'hypothèse de Marc Angenot est que cette atteinte ainsi portée à la littérature

21. Cr., de Dujardin, Les Lauriers sont coupés, anticipant la technique joycienne du monologue intérieur.

22. "Ceci tuera cela, ou: la chose imprimée contre le livre ", dans Romantisme, 44, 1984, 
par le journalisme, et au livre par le journal a déterminé chez les écrivains du temps et, singulièrement, chez les poètes (les plus enclins à mythifier l'une et l'autre), des réactions de défense et de repli, exprimées dans le lancement de programmes esthétiques rompant à tous égards avec le discours impérialiste et proliférant de la grande presse. Et de fait, dans la ligne de cette hypothèse, on constate que la référence au péril journalistique est récurrente dans les manifestes symbolistes et que la définition du langage poétique comme anticommunication, telle qu'elle est proposée par Mallarmé, ainsi que la doctrine de l'hermétisme qu'elle sous-tend et légitime, dépendent dans une large mesure (souvent méconnue) de cette rivalité inédite entre l'ordre littéraire et l'ordre médiatique. En restant à ce niveau d'analyse, on enregistre donc une fois de plus, comme dans les deux exemples déjà convoqués, un remodelage négatif, à valeur et fonction réactives (un discours se donne les moyens de barrer la mainmise de l'autre sur le champ discursif et préserve sa spécificité élective en accentuant ses marques). Cependant, à mieux y regarder, on peut relever que certains agents de la sphère restreinte adoptent, vis-à-vis de la presse, une position à la fois moins radicale et plus dialectique. Pour s'en tenir au cas le plus intéressant, celui de Mallarmé, on observe que son travail théorique, où s'agite inlassablement la question du "journal ", cherche les voies d'un dépassement à cette crise, non plus (ou pas seulement) en enjoignant le poète à distinguer sa pratique du discours médiatique mais bien en l'invitant à reprendre au journal son bien : à détourner, en somme, à des fins esthétiques ce qui, en celui-là, n'a d'autres fonctions que pragmatiques et informationnelles (par exemple, le pli libre de la feuille imprimée, la double et grande page, le jeu des divers corps et épaisseurs de caractères, etc.). Ce dont lui-même donnera, au terme de sa vie, l'exemple éloquent, avec Un Coup de dés, qui table sur un dispositif typographique foisonnant, où des phrases-titres, étalées à travers le "livre" et étagées à divers niveaux sur les pages, s'enlèvent en grandes capitales et en grasses sur le fond d'un texte-mosaïque ${ }^{23}$. Entreprise dont l'un des enjeux peu aperçu - relancer symboliquement le modèle journalistique - procède d'un fait complexe de transaction, opérant à la fois par assimilation et différenciation, et ouvrant la voie à maintes démarches avantgardistes du siècle suivant ${ }^{24}$.

\section{La (re)production symbolique des classes sociales}

Si les processus transactionnels, avec leur dialectique de la reprise et du rejet, ont retenu à travers ces exemples notre attention, c'est notamment parce

23. Pour des développements plus fournis sur le rapport de Mallarmé au péril médiatique et, plus largement, la relation nouée par le projet du Livre avec la crise fin-de-siècle, voir Pascal Durand, "Le Livre, instrument institutionnel. Mallarmé et la crise des valeurs symboliques", dans Charles yan Lerberghe et le symbolisme. Actes du colloque d'Aix-la-Chapelle, Presses de l'université de Cologne.

24. Voir P. Durand, "D'une rupture intégrante, avant-gardes et transactions symboliques", dans Pratiques, S0, juin 1986, p. 31-45. 
qu'ils font voir que, dans ou derrière le conflit des classes et des classements, se joue un conflit des imaginations formelles et thématiques (émergence de formes et de contenus "neufs" ou régressions réactionnaires) qui en est, semble-t-il, partiellement la cause et partiellement l'effet. Nous avons voulu de la sorte mettre en évidence le pouvoir du système à s'auto-constituer, à se produire et à se reproduire. Objectera-t-on qu'à notre insu, nous n'avons fait ainsi que transposer dans l'ordre des classes textuelles la logique systémique de l'ordre des genres, tel qu'il est pensé par les poéticiens ${ }^{25}$ ? Ce serait oublier que, comme nous l'avons suggéré tout au long, ces classes, en cela même qu'elles masquent leurs effets de division et de stratification, ne cessent d'être porteuses d'une signification sociale aussi occulte qu'agissante. Tel sera l'objet d'une prochaine recherche, de déterminer les points et modalités de rencontre entre ce sens social des classes textuelles et, d'une part, la configuration des instances collectives qui gèrent l'appareil littéraire (la socialité complice des acteurs du champ) et, d'autre part, l'économie générale de la société "réelle ".

Sauf à reconduire les illusions homologiques, cette même recherche ne pourra toutefois négliger le fait que, tout investies qu'elles soient par la socialité, les divisions symboliques ont sans doute, comme nous l'avons soutenu, leur part d'autonomie mais qu'en outre elles sont virtuellement capables, à l'intérieur d'un dispositif culturel aussi structuré que la littérature, de produire en retour des effets de construction du social, soit fictifs, soit effectifs. Tel ensemble de textes peut, ainsi, viser ou plutôt susciter une classe de lecteurs en porte à faux sur deux ou plusieurs fractions sociales, lecteurs socialement disparates, unis par une connivence seconde qui serait, en quelque sorte, leur conscience (esthétique) de classe.

La littérature n'est pas seulement devant (ou dans) la société comme une instance recevant servilement de celle-ci des impulsions déterminantes qu'elle traduit en classements symboliques; elle est aussi, vis-à-vis de la formation sociale, en position d'analyseur, la découpant et la ré-articulant selon sa logique. Avec elle, tout à la fois domaine et instrument d'analyse, les sociologues détiennent peut-être, l'un des outils les plus efficaces dont ils puissent user dans leur démarche critique. Sans aller jusqu'à soutenir, avec Mallarmé, que "le rapport social et sa mesure momentanée qu'on la serre ou l'allonge [sont] une fiction, laquelle relève des belles lettres ${ }^{26} "$, on peut estimer qu'ils ont notamment à apprendre, de celles-ci, que le symbolique inlassablement travaille le social.

25. C'est ici le lieu d'indiquer que si l'exposé qui précède a, plus d'une fois, donné l'impression de fonder entièrement la hiérarchie des classes en fonction de la distribution des genres, cette procédure imposée par la commodité méthodologique n'exclut en rien qu'une recherche plus détaillée et plus construite prenne en compte des critères classificatoires autres que génériques. Ainsi il est évident que certains labels auctoriaux (le roman-Duras et à la Duras, le roman-Guy des Cars) ou éditoriaux (le roman-Minuit et le roman-J'ai lu) fonctionnent comme des classes à part entière. Dans le cas particulier de * J'ai lu n, on remarquera que son effet de classe est notamment d'homogénéiser des textes préalablement publiés chez d'autres éditeurs ou en d'autres collections.

26. Mallarmé, "Sauvegarde", dans Euvres complètes, éd. cit., p. 420. 